\title{
Multimodales Erzählen in der Orchesterprobe
}

\author{
Monika Messner (Salzburg)
}

\begin{abstract}
The focus of attention in this contribution are narrative sequences in orchestra rehearsals realized by the conductor using different semiotic resources such as talk, gesture, facial expressions, gaze and body movement. Adopting a conversation analytic approach to a video-recorded orchestra rehearsal, the article aims to illustrate how narrative elements are implemented in the primarily instructive context of the rehearsal and which functions such narrations fulfil (i. e. joke, argumentation etc.). It will be demonstrated how storytelling is started, unfolded and brought to an end under sequential and multimodal conditions. The analysis also considers how the musicians react to the conductor's narrations - through gaze, facial expressions and talk and how they contribute as listeners to the production of narratives. The findings show that the conductor as the narrator and the musicians as his audience organize narrating in orchestra rehearsals in an interactive, multimodal and sequential way.
\end{abstract}

\section{$1 \quad$ Einleitung}

Eine Orchesterprobe ist vor allem - aber nicht ausschließlich - geprägt durch Anweisungen und Korrekturen des/der Dirigenten/in, die er/sie zur Performanz der Musiker/innen gibt. Für solche Anweisungen stehen ihm/ihr sowohl verbale als auch nonverbale Ausdrucksmittel zur Verfügung, wie Gestik, Mimik, Körper- und Kopfbewegungen, Blicke etc. Die Orchesterprobe ist ein Beispiel des sog. „Sprechen neben Handeln“ (cf. „,coordinated task activity“ nach Goffman 1979:16), wo in einer Interaktionssituation nicht das Sprechen im Zentrum steht, sondern eine gemeinsam betriebene Aktivität, die durch das Sprechen kommentiert, strukturiert und/oder gemanagt wird. Die Musiker/innen antworten musikalisch auf die Anweisungen des/der Dirigenten/in und zeigen damit auf, wie sie die vorausgegangenen Ansagen verstanden haben. Auf diese Weise entsteht in der Orchesterprobe Interaktivität.

Es kann aber auch durchaus vorkommen, dass der/die Dirigent/in in seine/ihre Anweisungen Erzählstrukturen einbettet, die er/sie multimodal realisiert. Multimodal bedeutet, dass Ressourcen unterschiedlicher Modalitäten eingesetzt werden, etwa Vokalität, Gestik, Mimik, bestimmte Körperhaltungen, Bewegungen im Raum oder auch die Manipulation von Objekten (cf. Deppermann 2018). Der/die Dirigent/in kann etwa Hintergründe zum gerade gespielten Werk, vergangene Erfahrungen mit dem Werk oder dem Komponisten, sowie eigene Vorstellungen zum Werk narrativ illustrieren bzw. rekonstruieren. Auf diese narrativen Strukturen können die Musiker/innen wiederum musikalisch antworten. Daneben können sie auch nichtmusikalisch, z. B. durch ein Nicken, darauf reagieren und dadurch anzuzeigen, dass sie die 
Geschichte des/der Dirigenten/in würdigen oder verstanden haben - so wie es z. B. in einem Alltagsgespräch vorkommen kann. Dieser Beitrag setzt sich zum Ziel, die Interaktion zwischen Dirigent/Dirigentin und Orchester bezüglich solcher narrativer Sequenzen zu beschreiben, insbesondere in ihrer multimodalen Ausprägung.

Dabei wird Erzählen als Praktik verstanden, mit der vergangene Ereignisse und Erfahrungen kommunikativ rekonstruiert werden. Erzählen gehört zu den rekonstruktiven Gattungen und erfüllt verschiedene Funktionen in der Lebenswelt der Sprecher/innen (cf. Bergmann/Luckmann 1995). Narrative Rekonstruktionen können etwa Beschwerden oder Rechtfertigungen zum Ausdruck bringen, sie können zur Erheiterung oder Auflockerung eingesetzt werden oder zuvor geäußerte Behauptungen explizieren (cf. Mandelbaum 2013; König/Oloff 2018a).

In der Forschung zu Erzählstrukturen wird als zentrales Merkmal - v. a. im Hinblick auf Alltagsgespräche - die vorübergehende Auflösung der Sprecherwechselsystematik (Sacks/Schegloff/Jefferson 1974) gehandelt. Zu Beginn einer Erzählung, die in ein Alltagsgespräch integriert ist, werden die bis dato bestehenden Sprecherwechselregeln kurzerhand außer Kraft gesetzt, damit ein Sprecher/eine Sprecherin eine Geschichte erzählen kann. Der/die Zuhörer/in kann im Laufe der Geschichte dem/der Sprecher/in durch Rezeptionssignale, wie z. B. Kopfnicken, kurze Kommentare, Bewertungen, etc. anzeigen, wie er/sie das Erzählte interpretiert. ${ }^{1}$ In der Orchesterprobe muss es jedoch gar nicht erst zur Auflösung der Sprecherwechselsystematik kommen, da das Rederecht asymmetrisch auf die Interaktionsteilnehmer/innen verteilt ist: Der/die Dirigent/in hat als Primus inter Pares das Recht, das Orchester an jeder beliebigen Stelle während der Probe zu unterbrechen, um verbale Anweisungen an die Musiker/innen zu richten. Außerdem bestimmt der/die Dirigent/in, wann die Musiker/innen nach einer Unterbrechung wieder weiterspielen dürfen. Das verbale Rederecht ist somit weitestgehend auf den/die Dirigenten/in beschränkt.

Die Orchesterprobe zeichnet sich durch ein besonderes „participation framework“ (Goffman 1981; Goodwin/Goodwin 2004; Goodwin 2007) mit einer leitenden bzw. tonangebenden Person an der Spitze eines Kollektivs aus. Dieses Teilnehmer/innen-Format ist bereits räumlichstrukturell vorgegeben: Der/die Dirigent/in steht oder sitzt erhöht auf einem kleinen Podest vor den Musiker/inne/n, die Musiker/innen selbst sitzen in einem Halbkreis angeordnet mit Blick auf den/die Dirigenten/in. Der/die Dirigent/in mit seinem/ihrem (zumeist alleinigen) Rederecht kann auch kurze Geschichten ankündigen, einleiten und erzählen, ohne vorher einen turn-byturn-talk ${ }^{2}$ außer Kraft setzen zu müssen.

Aus diesen Überlegungen heraus untersucht der vorliegende Beitrag anhand empirischen Videomaterials, wo Erzählsequenzen in der Orchesterprobe auftauchen, wie sie in den Kontext der Orchesterprobe einbezogen sind und welche Funktion ihnen zukommt (etwa Auflockerung, Argumentation etc.). Außerdem wird gezeigt, wie die Musiker/innen - währenddessen oder auch

\footnotetext{
${ }^{1}$ Zu den interaktiven Aspekten des Erzählens cf. vor allem Quasthoff (1980), Schegloff (1997), Bonu (1998), Quasthoff (200) und Lucius-Hoene/Deppermann (2002) sowie Kapitel 2.1.

${ }^{2}$ Ein turn-by-turn-talk zeichnet sich durch Redebeiträge aus, die sequenziell und geordnet aufeinander folgen. Die Interaktanten/innen zeigen u. a. durch intonatorische, semantische und syntaktische Mittel an, wann ihr Turn abgeschlossen ist und ein Sprecherwechsel stattfinden kann (cf. dazu u. a. Sacks/Schegloff/Jefferson 1974; Gülich/Mondada 2008).
} 
danach - auf solche narrativen Rekonstruktionen reagieren und damit als Zuhörer/innen ebenfalls zum Erzählten beitragen (cf. „,coconstruction“ oder auch ,,joint construction“ bei Schegloff 1997: 97). Dabei sollen vor allem non-verbale Strukturen herausgearbeitet werden, da sie - wie bereits weiter oben angeführt - in der Orchesterprobe eine wesentliche Rolle spielen. Die vorliegende Studie setzt sich zum Ziel, anhand der Untersuchung eines spezifischen institutionellen Kontexts, neue Perspektiven auf die vielfältigen Funktionen des multimodalen Erzählens in der sozialen Interaktion zu eröffnen.

Multimodales Erzählen im spezifischen institutionellen Kontext der Orchesterprobe blieb bisher unerforscht. Generell gibt es wenige Studien, die den Einsatz multimodaler Ressourcen während des Erzählens fokussieren (König/Oloff 2018a: 220). Da Erzählen allerdings in zeitlich-räumlicher Ko-Präsenz der Beteiligten stattfindet und gleichzeitig den Einsatz von körperlichen und materiellen Ressourcen ermöglicht, wird es zunehmend als „körpergebundene und verkörperlichte Praktik“ (König/Oloff 2018b: 279) verstanden. In dieses Plädoyer für eine Konzeptualisierung von Erzählen als multimodal realisierte Aktivität (König/Oloff 2018a: 215) soll sich auch der vorliegende Beitrag einordnen und untersuchen, wie unterschiedliche kinesische Ressourcen (etwa Mimik, Blick, Kopfbewegungen, Gestik, Handhabung von Objekten, Proxemik und Körperbewegungen) zum Zweck des Erzählens eingesetzt werden.

Das methodische Vorgehen in der Untersuchung der für diese Studie zugrundeliegenden Daten ist grundsätzlich gesprächsanalytisch orientiert (Deppermann 2008). Auf diese Weise kann sequenzanalytisch gezeigt werden, wie Erzählaktivitäten in den Gesprächskontext, den sequenziellen Verlauf und in die Interaktion zwischen dem/der Dirigenten/in als Erzähler/in und den Musiker/inne/n als Zuhörer/innen eingebettet sind.

Im vorliegenden Beitrag wird in Abschnitt 2 der theoretische Rahmen abgesteckt, der hinsichtlich der Relevanz für die zu beantwortenden Fragen sowie für die Daten, (interaktives) Erzählen und Multimodalität (v. a. in der Orchesterprobe) in den Mittelpunkt stellt. Dabei wird zuerst auf die Erforschung von Erzählstrukturen in gesprächsanalytischer Perspektive eingegangen, bevor der Fokus auf Erzählen in institutionellen Settings und nicht zuletzt auf die Auseinandersetzung mit dem multimodalen Charakter von Orchesterproben gelegt wird. Daran anschließend wird in Abschnitt 3 das Datencorpus für die vorliegende Studie erläutert. Der Abschnitt 4 präsentiert die Analyse anhand eines Transkriptausschnitts, in dem der Dirigent etwas über eine nicht anwesende Person (einen Pianisten) erzählt. Im abschließenden Fazit (Abschnitt 6) werden die Ergebnisse herausgestellt. Es kann zum einen gezeigt werden, dass sich das Erzählen aus instruierenden Handlungen des/der Dirigenten/in heraus entwickelt, zum anderen, dass narrative Rekonstruktionen in Orchesterproben interaktiv, sequenziell und multimodal realisiert werden.

\section{Zur Spezifik des (interaktiven) Erzählens und der Multimodalität in Orchester- proben}

\subsection{Die Erforschung von Erzählstrukturen in gesprächsanalytischer Perspektive}

Die konversationelle Erzählforschung geht auf Harvey Sacks zurück, der mehrere seiner Lectures dem Erzählen widmete, wie insbesondere Band II der beiden von Gail Jefferson heraus- 
gegebenen Sammelbände dokumentiert (cf. Sacks 1992). Die konversationsanalytische Forschung zu Erzählaktivitäten charakterisiert diese v. a. durch die Unterbrechung der ansonsten üblichen turn-by-turn-Ordnung zugunsten eines/einer nun länger am Wort bleibenden Sprechers/in. Das Interesse von Sacks an Erzählungen hat sich aus der Beschäftigung mit der Sprecherwechsel-Organisation (turn taking ${ }^{3}$ ) heraus entwickelt. Dabei steht v. a. die Frage im Vordergrund, wie es angesichts der Regeln für die Verteilung des Rederechts überhaupt dazu kommt, dass Sprecher/innen längere Redebeiträge für sich beanspruchen können, ohne von anderen Gesprächsteilnehmern/innen unterbrochen zu werden.

Sacks (1992: 18) stellt fest, dass ein/e Sprecher/in seinem/ihrem Gegenüber durch ein „story preface" zu verstehen geben kann, dass nun ein längerer und komplexerer Beitrag folgen und die Sprecherwechselsystematik vorübergehend außer Kraft gesetzt wird. „Story prefaces“ oder Geschichtseinleitungen leiten nach Sacks $(1971,1989,1992)$ nicht nur eine Erzählung ein, sondern klären auch den Bezug zum Thema der Interaktion, die Rolle des/der Erzählers/in darin und seine/ihre Haltung zu Erzählung. Auf diese Einleitung folgt nach Jefferson (1978: 219) nochmals ein turn des Gegenübers, in dem er/sie sich als „story recipient“" positioniert. Dann zeichnet sich die eigentliche Erzählung ab, die meistens in einem Höhepunkt gipfelt, bevor eine erneute Rückkehr in den turn-by-turn-talk erfolgt und die Sprecherwechsel-Ordnung wieder aufgenommen wird. Das Ende einer Geschichte wird nach Sacks (1989) durch eine Antwortoder Bearbeitungssequenz komplettiert, in der die Zuhörer/innen auf die Geschichte reagieren und sie auch kommentieren.

Aus diesen Überlegungen geht hervor, dass nicht nur der/die Erzähler/in während einer narrativen Sequenz wichtig ist, sondern auch den Zuhörern/innen eine wesentliche Rolle zukommt. Denn sie sind es, die dem/der Erzähler/in anzeigen, ob und wie sie die Geschichte verstanden haben und ob sie letztere auch würdigen. Sacks stellt bereits in seinen Vorlesungen 1971 fest, dass

„part of the common business of story-telling occasions involves story recipients positioning an appreciation of the story on its completion. So that it's an altogether common feature of storytelling occasions that, on some story's recognized completion, recipients will offer understandings of the story."

(Sacks 1971: 7)

Dieser interaktive Aspekt des Erzählens rückt im Anschluss an Sacks immer stärker in den Mittelpunkt der Erzählforschung (cf. u. a. Jefferson 1978; Quasthoff 1980, 2001; Goodwin 1984; Schegloff 1997; Bonu 1998; Lucius-Hoene/Deppermann 2002; Quasthoff/Becker 2005; De Fina/Georgakopoulou 2012, 2015; Kotthoff 2017). Die Hervorbringung von Erzählstrukturen wird dabei als interaktive Leistung aller Interaktionsteilnehmer/innen beschrieben. ${ }^{4}$ Die Beteiligten können sich auf unterschiedliche Weise in die Erzählung einbringen: als Erzähler/in, als Ko-Erzähler/in oder als Zuhörer/in. Diese sog. „participant roles“ werden dynamisch und

\footnotetext{
${ }^{3}$ Das turn taking (oder der Sprecherwechsel) reguliert die Verteilung des Rederechts in einem Gespräch. An einer übergaberelevanten Stelle eines Gesprächsbeitrags kann ein Sprecherwechsel dadurch erfolgen, dass entweder der/die aktuelle Sprecher/in den/die nächste/n Sprecher/in auswählt, sich ein/e nächste/r Sprecher/in selbst auswählt, oder der/die aktuelle Sprecher/in weiterspricht (cf. Sacks/Schegloff/Jefferson 1974).

${ }^{4} \mathrm{Cf}$. auch Mandelbaum (2013: 501): ,[t]he 'audience' is in fact the co-author [...] with recipient turns playing a crucial role in shaping and even constituting the ongoing course of the storytelling".
} 
lokal ausgehandelt (cf. Schwarze 2019). Interaktiv ist Erzählen auch deshalb, da Erzähler/innen stets das (vermutete) Vorwissen der Zuhörer/innen - im Sinne eines Adressatenzuschnitts (,recipient design“" nach Sacks/Schegloff/Jefferson 1974: 727) - mit einbeziehen (cf. König/Oloff 2018b). Erzählungen werden je nach Adressat/in unterschiedlich gestaltet, wobei nicht nur sprachliche, sondern auch stimmliche und körperlich-räumliche Ressourcen genutzt werden (cf. Gülich 2008; König/Oloff 2018b).

Diese „Gestaltungsorientiertheit“ (Gülich 2008: 409) geht auch einher mit der Haltung (,stance"), die Erzähler/innen in Bezug auf ihre Geschichte einnehmen können (cf. Stivers 2008; Niemelä 2010, 2011). Durch die Einnahme einer bestimmten Haltung geben die Erzählenden ihrem Publikum bereits vor, welche Art von Reaktion sie sich erwarten: wird eine spaBige Geschichte erzählt, so soll gelacht werden, wird eine traurige Geschichte erzählt, wird eine empathische Reaktion angestrebt etc. Die Zuhörer/innen können die Haltung des/der Erzählers/in teilen und bestätigen (affiliation) oder aber auch ablehnen. Stivers (2008) und Niemelä (2011) sprechen von ,,alignment“", insofern sich die Interaktanten/innen gemeinsam auf die konversationelle Aktivität, nämlich das Erzählen, ausrichten. Affiliativ ist ein/e Rezipient/in darüber hinaus, wenn er/sie dieselbe Haltung zum Erzählten einnimmt wie der/die Geschichtenerzähler/in selbst (siehe ,a like-minded stand on the content“" nach Niemelä 2011: 18; cf. auch Steensig 2013).

Zuhörer/innen können sowohl während des Erzählprozesses als auch im Anschluss an die eigentliche Erzählung durch vokale Reaktionen (etwa Lachen, Stöhnen), durch verbale Kommentare, durch Blickverhalten und durch Gestik (etwa Nicken) das Erzählte bearbeiten und gratifizieren (cf. Goodwin 1984; Gülich 2008). Erzählen wird damit interaktiv und ko-konstruiert von Sprecher/in und Hörer/in realisiert, es gibt in keinem Fall einen „einsamen Erzähler“ (Kallmeyer 1981: 420).

\subsection{Erzählen in institutionellen Settings}

Gülich (2004) unterscheidet hinsichtlich unterschiedlicher Interaktionssituationen und Kontexte verschiedene Typen von Erzählungen, wie z. B. alltägliches Erzählen, Erzählen während einer Krankenhausvisite, Erzählen in der Sozialberatung, in der Schule, usf. Insgesamt kann eine Einteilung in alltägliches und institutionelles Erzählen erfolgen.

Hinsichtlich Erzählungen in Institutionen gibt es Untersuchungen aus erzähldidaktischer Perspektive (cf. z. B. Ehlich 1984; Hausendorf/Quasthoff 1996; Becker-Mrotzek 2011; Ohlus 2014, 2016), die sich vor allem mit dem Erzählen von Geschichten im Unterricht auseinandersetzen. Die Kommunikation in der Schule wurde auch hinsichtlich interaktional hervorgebrachter Erzählschemata in Sprechstundengesprächen bzw. Elternsprechtagsgesprächen untersucht (cf. Kotthoff 2015, 2018). Außerdem befasst sich die Erzählforschung mit Erzählungen und Techniken der narrativen Rekonstruktion im Arzt-Patient-Gespräch (cf. z. B. Martens-Schmid 2000; Gülich/Schöndienst/Surmann 2003; Birkner 2017; Kotthoff 2018), oder auch mit Erzählstrukturen von Immigranten bei Sozialberatungen (cf. Rehbein 1980; Quasthoff 1980). Nicht zuletzt gibt es Untersuchungen zu narrativen Sequenzen vor Gericht (cf. Leodolter 1975; Hoffmann 1980) sowie zu massenmedialem Erzählen in einer TV-Unterhaltungssendung (Oloff/König 2018). 
Für den vorliegenden Beitrag ist vor allem die Studie von Ohlus (2016) interessant. Der Autor erarbeitet mündliche Erzählungen in einem pädagogischen Kontext unter den Aspekten Interaktivität, Prozessualität und Multimodalität und stellt dabei fest, dass unterschiedliche semiotische Ressourcen eine zentrale Rolle in der narrativen Rekonstruktion spielen. Während die Erzählerin auf verbaler Ebene ein klar elaborierendes Diskursmuster (Hausendorf/Quasthoff 1996) verfolgt und sich damit auch narrativ zum Geschehen distanziert, setzt sie ikonische Gesten zur Dramatisierung und zur Inszenierung ein. Auf ähnliche Art und Weise soll im vorliegenden Beitrag untersucht werden, wie Erzählprozess, Erzählstruktur und Interaktion in narrativen Rekonstruktionen in der Orchesterprobe zusammenhängen und wie diese durch den Einsatz verschiedener semiotischer Ressourcen beeinflusst werden.

Daneben beschreibt Birkner (2017) Erzählungen in Arzt-Patienten-Gesprächen als Veranschaulichungsverfahren und grenzt diese gegenüber ausgebauten Alltagserzählungen (sog. ,,big packages “, cf. Sacks 1992: 354-359) ab. Solche narrativen Illustrationen, die beispielsweise von Patienten/innen eingesetzt werden, um Beschwerden zu artikulieren, fallen unter sog. „small stories" (cf. Bamberg/Georgakopoulou 2008) und sind in ihrer Funktion auf den institutionellen Kontext zugeschnitten (cf. auch Kotthoff 2018). Diese narrativen Kleinformate müssen nicht zwingend linear ablaufen oder abgeschlossen sein und können sogar lediglich aus Andeutungen von einer Erzählung bestehen (cf. Bamberg/Georgakopoulou 2008; König/Oloff 2018a). Kotthoff (2018: 245) hält zu narrativen Fragmenten Folgendes fest:

In der Regel werden Erzählungen dann nicht ausgebaut, wenn sie sich in eine übergeordnete Aktivität einbetten, sei diese selbst [...] deskriptiv (wie in der Arztpraxis oder in diversen institutionellen Sprechstunden und Beratungen) oder argumentativ, wenn sie eine Position durch eine unvollständige Beleggeschichte unterfüttern sollen.

(Kotthoff 2018: 245)

Auch in der Orchesterprobe stehen Erzählungen häufig im Dienst des institutionellen Kontexts und dienen zur Veranschaulichung oder Erklärung von bestimmten Sachverhalten (z. B. eine bestimmte Spiel- oder Interpretationsweise). Dabei werden sie nicht als sequenziell komplexe soziale Handlungen realisiert, sondern bestehen aus Fragmenten und sind Teil von größeren Handlungen, allen voran jener des Instruierens.

In institutionellen Settings gelten aufgrund der spezifischen Rollenverteilung der Beteiligten interaktive Bedingungen, die von jenen eines alltäglichen Gesprächs abweichen (cf. Drew/Heritage 1992). In einem Alltagsgespräch ist nicht vorhersehbar, welche/r Sprecher/in wie viele Turns übernimmt, wer in welcher Abfolge das Rederecht erhält oder welche Themen sich im Laufe eines Gesprächs ergeben. In einer institutionellen Interaktion sind dahingegen bestimmte Aspekte von vornherein bereits definiert, z. B. erwartbare Themen oder die Rollenverteilung (cf. Sacher/Knerich/Lingnau 2015: 349). Dies gilt auch für Erzählungen und Geschichten, die Teil von Interaktionen in institutionellen Settings sein können.

Quasthoff (2001) benennt ein zentrales Merkmal von Erzählungen in institutionellen Settings, nämlich dass Vertreter/innen der Institution in professionellen Interaktionssituationen nur selten erzählen. Nach Quasthoff (ibd.) macht allenfalls der/die Klient/in einer Institution Gebrauch 
einer typischen Alltagserzählung, in die er/sie selbst involviert ist und die für ihn/sie erzählwürdig5 ist: Die Patienten/innen, die Schüler/innen, die Zeugen/innen, die Angeklagten, etc. Dieses Merkmal ist darauf zurückzuführen, dass in einer institutionellen Interaktionssituation die Beteiligten den professionellen Charakter des Gesprächs beibehalten möchten. Durch eine (allzu) persönliche Erzählung hingegen könnte ein professionelles Gespräch schnell in ein privates Gespräch ausarten, was von den Beteiligten meist als unangebracht erachtet wird (ibd.: 1304).

Gewisse Personen sind außerdem ausgenommen, insbesondere Lehrer/innen in Lehr-LernKontexten, die Erzählungen zum Illustrieren von Sachverhalten einsetzen, oder auch Verkäufer/innen in Verkaufsgesprächen, die dem/der Käufer/in eine Ware anhand von beispielhaften Erzählungen anpreisen möchten. In solchen Interaktionssituationen ist mit der Erzählung wenig oder keine Unterhaltung beabsichtigt, sondern sie wird in den Dienst einer anderen Kommunikationsabsicht gestellt (cf. Kotthoff 2018).

In der Orchesterprobe können Dirigenten/innen ebenfalls Erzählungen - wie bereits weiter oben beschrieben - zum Veranschaulichen bestimmter Spiel- oder Interpretationsweisen einsetzen, oder aber auch um Sympathie herzustellen bzw. das Gespräch mit den Musiker/inne/n auf eine persönlichere Ebene zu bringen.

\subsection{Die Untersuchung von Multimodalität in der Orchesterprobe}

Eine Orchesterprobe zeichnet sich durch ihren stark multimodalen Charakter aus. Die gesamte Interaktion zwischen Dirigent/in und Orchester ist multimodal organisiert: Die musikalischen Turns der Musiker/innen wechseln sich mit den multimodal gestalteten Turns des/der Dirigenten/in ab. In den Beiträgen der Dirigenten/innen kommen zahlreiche, unterschiedliche multimodale Ressourcen zum Einsatz, wie z. B. Vokalität (in Form von Sprache, Prosodie und Gesang), Gestik, Mimik, Blick, Positur und Körperbewegungen.

Für die Orchesterprobe gibt es eine Reihe an Arbeiten, die die multimodale Ausrichtung der Interaktion zwischen Dirigent/in und Musiker/inne/n und dabei vor allem das Sprechen neben Handeln von Dirigenten/innen untersuchen. Poggi (2002) erörtert die kommunikativen Funktionen des Blicks, der Kopfbewegungen und des Gesichtsausdruck des/der Dirigenten/in und entwickelt daraus ein Lexikon. Dabei unterscheidet sie zwischen semiotischen Ressourcen, die eingesetzt werden, um zu vermitteln, wer spielen soll (z. B. durch einen Blick zu den betreffenden Musiker/inne/n), wann gespielt werden soll (z. B. durch ein Hochziehen der Augenbrauen vor dem Start der Musik) und wie gespielt werden soll (z. B. bedeutet ein Stirnrunzeln, dass forte gespielt werden soll). In einer Studie zur multimodalen Kommunikation von Chorleitern/innen untersucht die Autorin (cf. Poggi 2011), ob gestische und mimische Signale in der Lage sind, Emotionen zu vermitteln, die musikalisch umgesetzt werden sollen, ob sie Informationen zu musikalischen Parametern liefern (etwa Klangfarbe, Rhythmus, Melodie, musikalische Struktur) und ob sie Rückmeldungen zur Performance zum Ausdruck bringen können.

\footnotetext{
${ }^{5}$ Zum Konzept der Erzählwürdigkeit (auch reportability oder tellability) cf. u. a. Labov/Waletzky (1967); Quasthoff (1980, 2001); Sacks (1992); Hausendorf/Quasthoff (1996); Lucius-Hoene/Deppermann (2002); Gülich (2008); Martínez (2017).
} 
Daneben versuchen Boyes-Bräm/Bräm (2004) aus spezifischen Gesten des/der Dirigenten/in potentielle lexikalische Bedeutungen abzuleiten. So schreiben sie beispielsweise Gesten, die Objekte manipulieren, die Eigenschaft zu, Informationen zu Klangqualität, Artikulation oder musikalischer Entwicklung zu übermitteln (eine nach oben gehaltene Hand auf Brusthöhe kann etwa ausdrücken, dass der Ton gehalten werden soll). Die Autoren stellen außerdem fest, dass solche expressiven Gesten von Dirigenten/innen polysemen Charakter haben und ihre spezifische Bedeutung daher nur abgeleitet werden kann, wenn sie in einem bestimmten Kontext, wie dem der Orchesterprobe, verwendet werden. Hier spielt das geteilte Wissen (Clark 1996) zwischen Dirigent/in und Orchester eine wesentliche Rolle: „[t]he special derived meaning of these gestures [...] is only interpretable to persons who know the second target domain of these gestural metaphors, the playing of classical orchestral music“ (Boyes Bräm/Bräm 2000: 166).

In einer ethnographisch ausgerichteten Studie untersucht Weeks (1996) Korrekturen und Instruktionen in einer Probe eines Amateurorchesters und erarbeitet bestimmte Techniken, die von Dirigenten/innen für korrigierende Aktivitäten eingesetzt werden. Er unterscheidet zwischen verbalen Instruktionen (etwa Erklärungen zu gewünschten musikalischen Effekten) und sog. ,illustrative expressions“ (Weeks 1996: 254). Darunter fallen Ausdrucksformen wie Singen, Sprechgesang und (Mit-)Zählen, mit denen - neben Sprache - korrigiert und instruiert werden kann. Auch wenn Weeks ausschließlich mit Audio-Daten arbeitet, stellt er trotzdem fest, dass solche vokalen Illustrierungen in Verbindung mit Gestik auftreten können, z. B. wenn der Dirigent eine bestimmte Stelle in der Partitur vorsingt und dabei mit den Armen und Händen einen Bogenstrich imitiert.

Veronesi (2014) ergründet darüber hinaus, wie es ein Lexikon an ideographischen Zeichen und Gesten, ${ }^{6}$ in Verbindung mit Sprache, dem Dirigenten ermöglicht, Korrekturen zum Spielen der Musiker/innen anzubringen und sequentiell zu organisieren. Ein weiteres Beispiel in Bezug auf das Zusammenwirken von verbalen und multimodalen Mitteln in der Probe liefert Merlino (2014): Sie setzt sich mit der Korrektur von Aussprachefehlern in Chorproben auseinander und zeigt, wie derartige Korrektur-Sequenzen in das komplexe Gefüge von multiplen Ressourcen, u. a. Sprache, Singen, Gestik, Körperausrichtung und Artefakte, eingebettet sind.

Nicht zuletzt zeigt Haviland (2007), wie in einer musikalischen Masterklasse für Streichinstrumente durch die Verwendung multimodaler interaktionaler Ressourcen Bedeutung dynamisch hergestellt wird. Dabei hebt er die Bedeutsamkeit des Körpers hervor, um etwa zu demonstrieren, wie eine musikalische Passage auf einem Streichinstrument gespielt werden soll: ,[b]odies [...] become at once vehicles of performance and meta-performance, means for making music and for communicating about music" (Haviland 2007: 170-171).

\footnotetext{
${ }^{6}$ Dieses Lexikon wird unter dem Begriff „Conduction ${ }^{\circledR}$ “ zusammengefasst. Es wurde von dem US-amerikanischen Dirigenten, Komponist und Arrangeur Lawrence D. „Butch“ Morris (1947-2013) ins Leben gerufen und bezeichnet ein Inventar an 55 Dirigiergesten, anhand derer der/die Dirigent/in Harmonie, Melodie, Rhythmus, Tempo, Artikulation, Phrasen u. Ä. an die Musiker/innen vermitteln kann, ohne dabei Noten oder eine Partitur zur Hilfe zu nehmen (cf. Morris 1995, 2006).
} 
In Bezug auf die oben genannten Studien stellt der vorliegende Beitrag das multimodale Erzählen in der Orchesterprobe in den Vordergrund und untersucht, wie dabei unterschiedliche semiotische Ressourcen ineinander wirken und wozu diese eingesetzt werden (z. B. zur Hervorhebung, zur Veranschaulichung, zur Erklärung etc.).

\section{Das Datencorpus}

Das Corpus für diese Arbeit besteht aus Videodaten, die Teil eines Dissertationsprojekts zur Interaktion zwischen Dirigent/in und Musiker/inne/n in Proben von französischen und italienischen Symphonie-Orchestern sind. Insgesamt umfasst das Corpus rund 55h Datenmaterial. Für die vorliegende Studie wurde ein Probenausschnitt von einem in Italien angesiedelten Orchester ausgesucht, in dem narrative Sequenzen vorkommen. In dem Beispiel ist die Erzählung in eine Instruktionssequenz eingebettet: Der Dirigent instruiert ein spezifisches Tempo und verknüpft diese Anweisung mit einem Narrativ, dem eine bestimmte Funktion zukommt. Analyseleitende Fragen sind die nach der Art der (multimodalen) Gestaltung der Erzählung, nach deren Verwobenheit mit instruktiven Elementen sowie nach deren Funktion im instruktionsspezifischen Kontext der Orchesterprobe. Die Transkriptionskonventionen folgen GAT II (Selting et al. 2009) für das Verbale, Nonverbales wird nach den Konventionen von Mondada (2014a) transkribiert.

\section{Narrative Sequenzen in Orchesterproben: „Il pianista maturizzato"}

Die folgende Analyse eines ausgewählten narrativen Verfahrens in einer Orchesterprobe soll zeigen, wie Dirigenten/innen Erzählungen multimodal ausgestalten und wie sich auch das Orchester durch verbale und nonverbale Reaktionen an deren Hervorbringung beteiligen kann. Ziel der Analyse ist es, multimodale Gestalten (Mondada 2014b, 2016) zu identifizieren.

Im folgenden Beispiel probt das Orchester das Concerto Nr. 1 in Do minore von Šostakovič, ein Konzert für Solo-Klavier, Solo-Trompete und Streichorchester. In diesem Beispiel probt nur das Orchester mit dem Dirigenten, ohne Solo-Instrumente. Nach einigen Korrekturen, die sich auf bereits Gespieltes beziehen, erzählt der Dirigent etwas über eine andere, nicht anwesende Person, nämlich einen Pianisten, den das Orchester schon kennt und mit dem sie bereits ein Solo-Werk für Klavier aufgeführt haben.

Im Transkript sind alle Interaktionsbeteiligten mit Kürzel versehen: der Dirigent (D), die Musiker/innen (MM), der Konzertmeister (KM), der Violinist, der direkt hinter dem Konzertmeister sitzt (V1), ein Violinist in der ersten Reihe (V2), sowie die Bratschistin, die rechts ganz außen in der ersten Reihe sitzt (B).

Beispiel 1: Il pianista maturizzato

Aufnahme_2_20072016, 0000

03:09-04:05

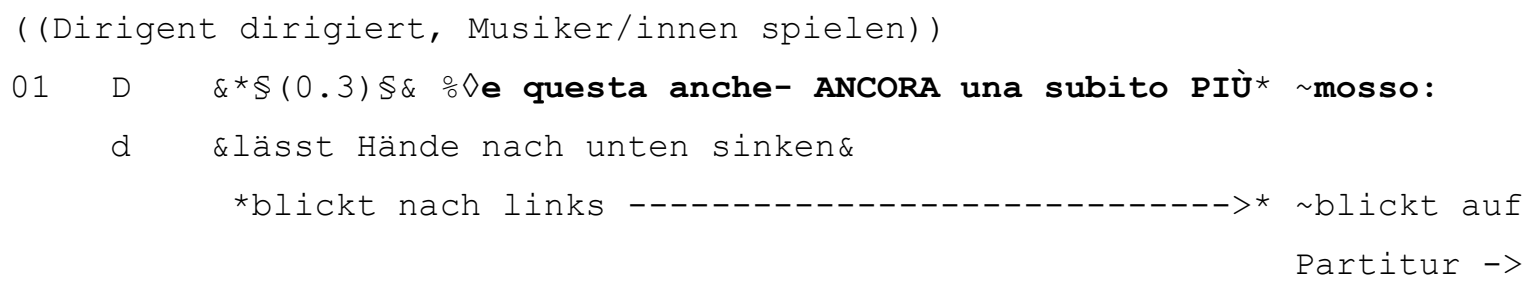




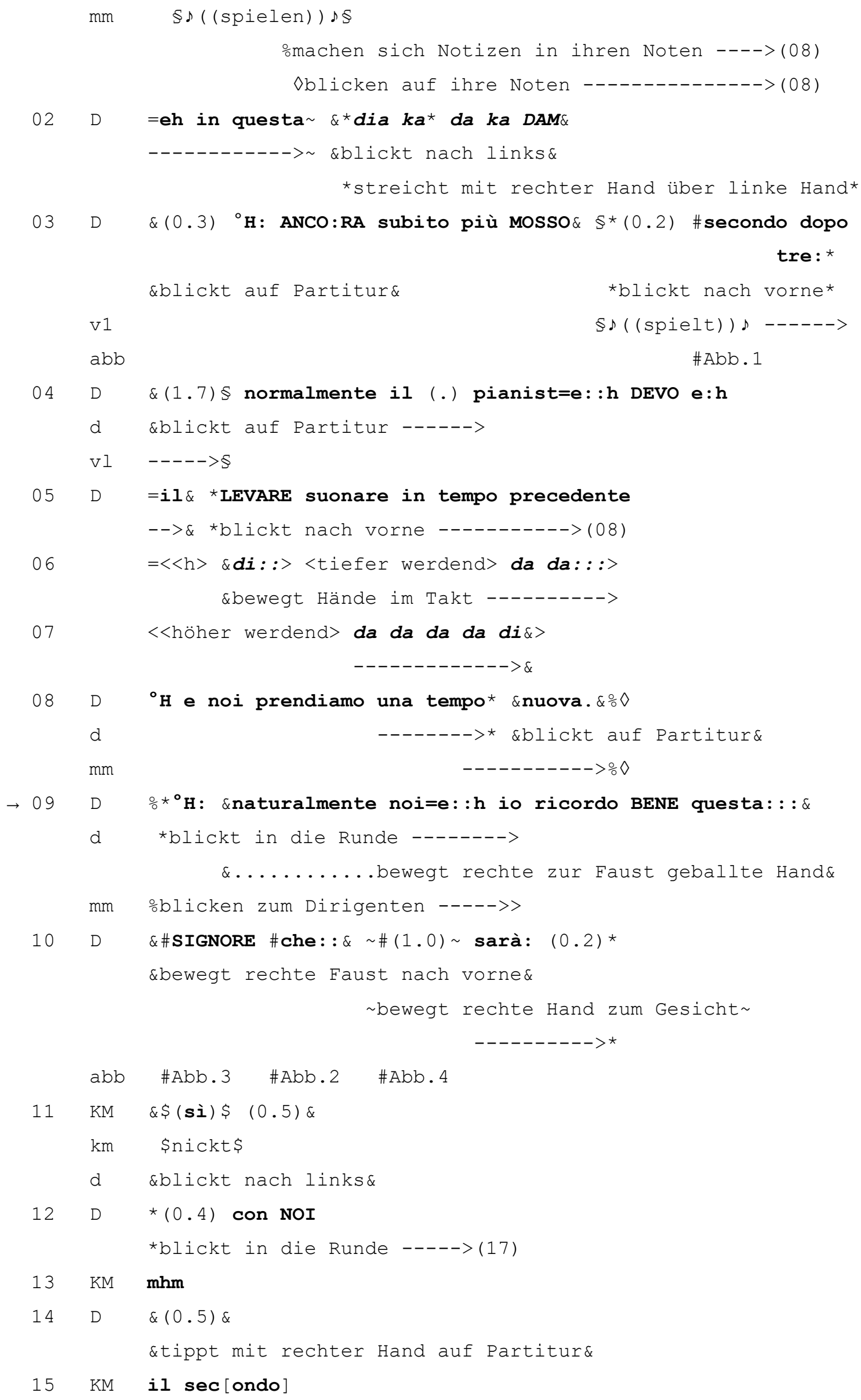




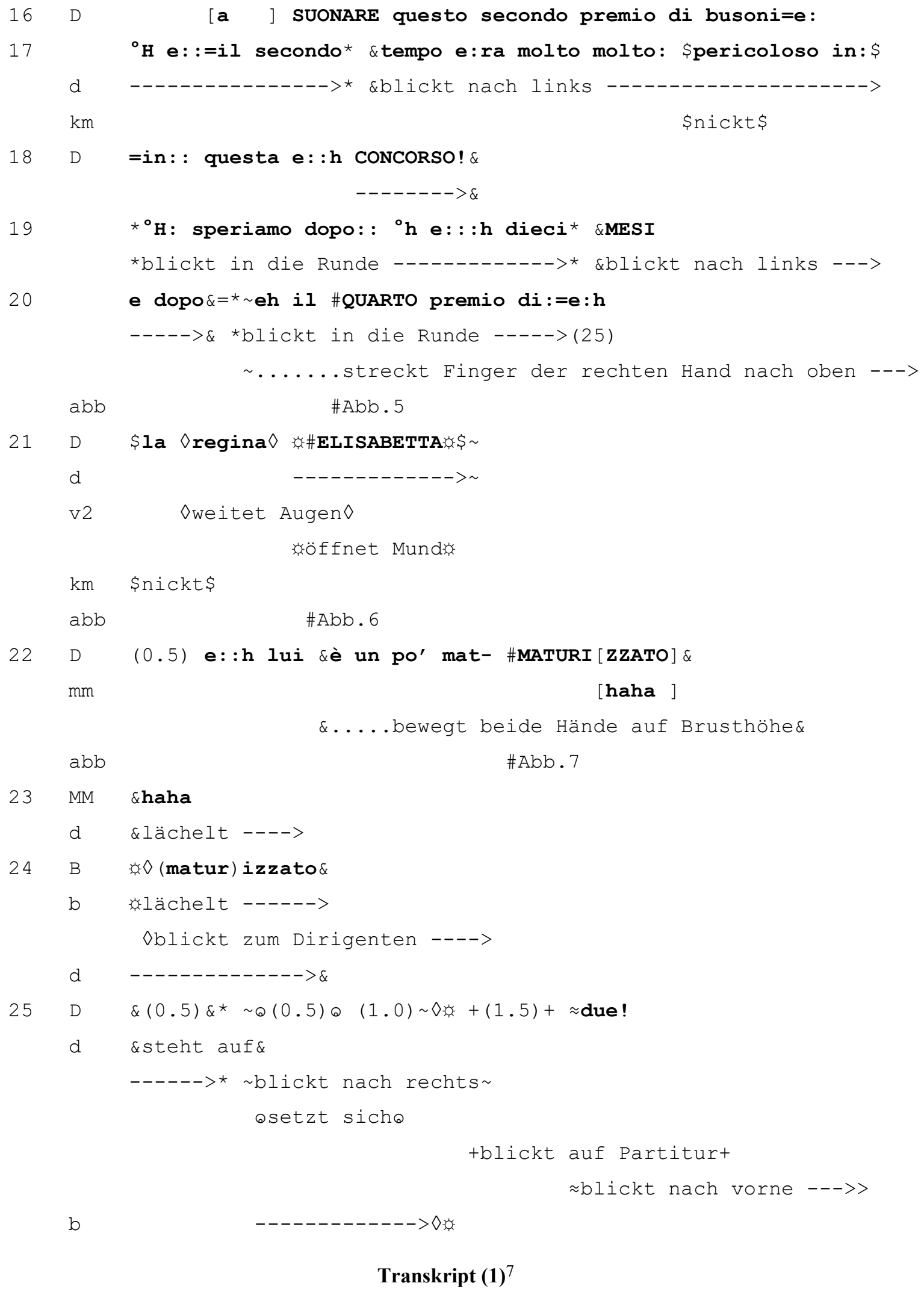

\section{Transkript (1) ${ }^{7}$}

Der Dirigent unterbricht die Musik in Z01 dadurch, dass er die Hände (und Arme) aus seiner Dirigierbewegung heraus nach unten sinken lässt und sodann nach links blickt, in die Richtung

\footnotetext{
${ }^{7} 01$, und diese auch NOCH eine subito più mosso'(subito più mosso = etwa: ,plötzlich bewegter') 02 ,äh in dieser dia ka da ka DAM'
} 
der Violinen. Es folgt eine Anweisung (ebenfalls in Z01), in der der Dirigent durch das Demonstrativpronomen questa anzeigt, dass er damit die unmittelbar vorher gespielte Stelle meint. Durch den Blick zu den Violinen gibt er zu verstehen, dass die Anmerkung vor allem an das Violinenregister gerichtet ist. Er singt die Stelle in Z02 (dia ka da ka dam) auch nochmals vor, zum einen, um die Stelle genauer einzugrenzen, zum anderen aber auch, um den Musiker/inne/n eine Vorstellung davon zu geben, wie die betreffende Stelle zu spielen ist. Gleichzeitig streicht der Dirigent, während er die Silben dia und $k a$ artikuliert, mit der rechten Hand über die linke Hand. Diese Geste erinnert stark an den Bogenstrich der Violinen. Beide Modalitäten - Gesang und Gestik - veranschaulichen hier zusätzlich zur verbalen Anweisung auf instruktive Art und Weise, was der Dirigent von den Musiker/inne/n hören möchte. In Z03 wiederholt der Dirigent die verbale Instruktion aus Z01, allerdings mit unterschiedlicher prosodischer Hervorhebung8 (ANCORA und PIU் in Z01, ANCORA und MOSSO in Z03) und komplettiert sie durch eine erneute Lokalisierung (secondo dopo tre). Allerdings blickt er hier zu allen Musiker/inne/n und nicht wie vorher allein zu den Violinist/inn/en. Während dieser verbalen Lokalisierung spielt der Violinist, der direkt hinter dem Konzertmeister sitzt, einige Töne auf seinem Instrument. Dieser musikalische Einwurf bleibt unkommentiert vom Dirigenten. Dieser wechselt - begleitet von einem Blick auf die Partitur - in Z04 zu einem Kommentar über den Pianisten, der in dem gerade gespielten Werk als Solist auftreten wird. In Z05-08 weist der Dirigent sowohl verbal, als auch gesanglich und gestisch an, wie die Stelle gemeinsam mit dem Pianisten zu spielen ist, sobald dieser auch in den Proben mit anwesend sein wird. Der Dirigent schließt diese Anweisung durch einen Blick auf die Partitur sowie eine fallende Intonation in seiner Stimme in Z08 (noi prendiamo una tempo nuova) ab.

Ab Z09 beginnt die eigentliche Erzählsequenz. Auffallend ist, dass der Dirigent in Z01-08 den Blick abwechselnd hin zum Orchester, auf die Partitur oder nach links, in die Richtung der Violinen ausrichtet. Ab Z09 blickt er fast ausschließlich zum gesamten Orchester, außer in Z11, Z17-18 und Z19, wo sich der Konzertmeister als (potentieller) Gesprächspartner für den Dirigenten einschaltet, oder in Z25, nachdem die Bratschistin, die rechts ganz außen in der ersten

03 ,NOCH [mehr] subito più MOSSO zweiter [Takt] nach drei'
04 ,normalerweise MUSS der Pianist äh'
05 ,den AUFTAKT im vorherigen Tempo spielen'
08 ,und wir nehmen ein neues Tempo auf'
09 ,natürlich wir äh ich kann mich GUT erinnern an diesen'
10 ,[PIANISTEN] der'
11 ,ja’
12 ,mit UNS [spielen wird]'
15 ,der zweite'
16 ,um diesen zweiten Busoni-Preis zu SPIELEN'
17 ,und das zweite Tempo war sehr sehr gefährlich in’
18 ,in diesem äh WETTBEWERB'
19 ,hoffen wir, dass äh nach zehn MONATEN'
20 ,und nach äh dem VIERTEN Preis'
21 ,der äh Königin ELISABETH'
22 ,äh er ein bisschen REIFER ist'
25 ,zwei'
8 Siehe zur Praktik des Hervorhebens bzw. des Highlighting von relevanten Informationen auch Goodwin (1994). 
Reihe sitzt, auf die Erzählung des Dirigenten in Z24 verbal reagiert hat. Auch die Musiker/innen sind bis Z09 mit ihren Noten beschäftigt, ab dann richten sie ihre Aufmerksamkeit dem Dirigenten und seiner Narration zu.

In Z09 leitet der Dirigent - nach einem markanten Luftholen - eine Erzählung ein, die sich auf den Solo-Pianisten bezieht. In seinem „story preface“ (Sacks 1992) beginnt er mit dem Ausdruck naturalmente noi und nimmt dabei das Personalpronomen noi nochmals auf, das er kurz zuvor in Z08 bereits verwendet hat. Damit zeigt er an, dass er sich selbst und das Orchester als Kollektiv versteht, das gemeinsam an einem musikalischen Werk arbeitet. In dieser Einleitungssequenz ist der Dirigent nicht gezwungen, die Sprecherwechsel-Systematik außer Kraft zu setzen, da er in der Orchesterprobe generell derjenige ist, der das Rederecht innehat. Nach Sacks (1989: 340-342) ist eine solche ,preface sequence“ in Alltagsgesprächen immer auch dadurch gekennzeichnet, dass sich ein/e Sprecher/in als Erzähler/in ankündigt und damit den anderen Gesprächsteilnehmern/innen zu verstehen gibt, dass erst nach Abschluss der Erzählaktivität ein Sprecherwechsel erwartet oder initiiert wird. In der Orchesterprobe fällt dieser Teil der Einleitungssequenz weg, der Dirigent beginnt seine Erzählung aus der vorherigen Erwähnung des Pianisten heraus und kennzeichnet dadurch den Übergang zur Narration.

Bei den Musiker/inne/n ist dennoch eine Reaktion zu erkennen, die als Turn bezeichnet werden kann, in dem sie sich als Rezipienten/innen der Erzählung hervortun. Während die Musiker/innen - wie weiter oben beschrieben - bis Z09 noch damit beschäftigt sind, sich die Korrekturen des Dirigenten in ihre Noten einzutragen, mit dem Blick auf ihr Notenpult verhaftet (siehe Abbildung 1), richten die meisten von ihnen ab Z09, also ab der Einleitung der Erzählung, den Blick und ihre Aufmerksamkeit dem Dirigenten zu (siehe Abbidlung 2). Der Dirigent wird hier also durch die Reaktion der Musiker/innen in seiner Rolle als Erzählender bestätigt:

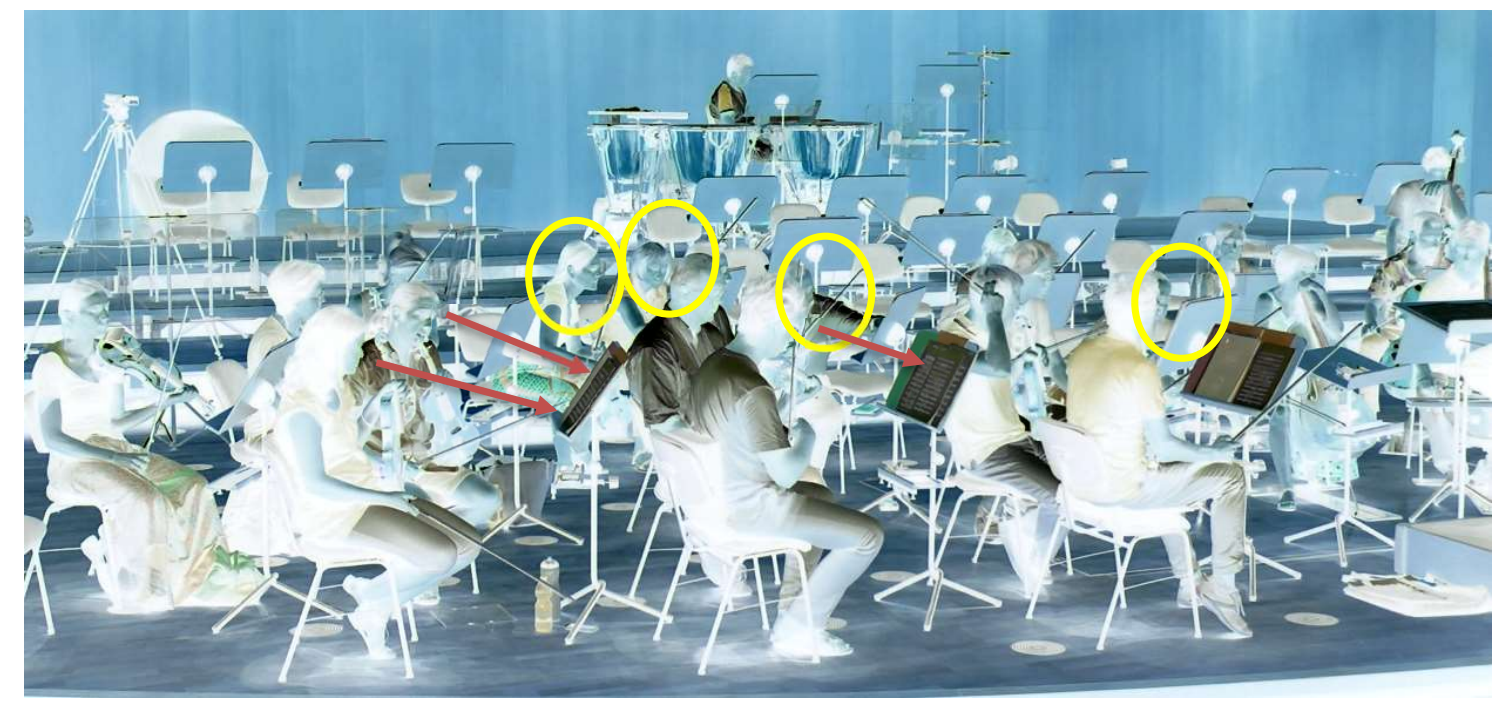

Abbildung 1: Aufmerksamkeit der Musiker/innen auf die Noten gerichtet (bis Z09) ${ }^{9}$

\footnotetext{
${ }^{9}$ Die gelben Kreise in der Abbildung zeigen die Musiker/innen, die sich Notizen in ihren Noten machen. Die Pfeile zeigen die Blickrichtung der Musiker/innen an (siehe auch Abbildung 2 und Abbildung 6). Bildrechte: Monika Messner; publiziert mit Einverständnis der abgebildeten Personen.
} 


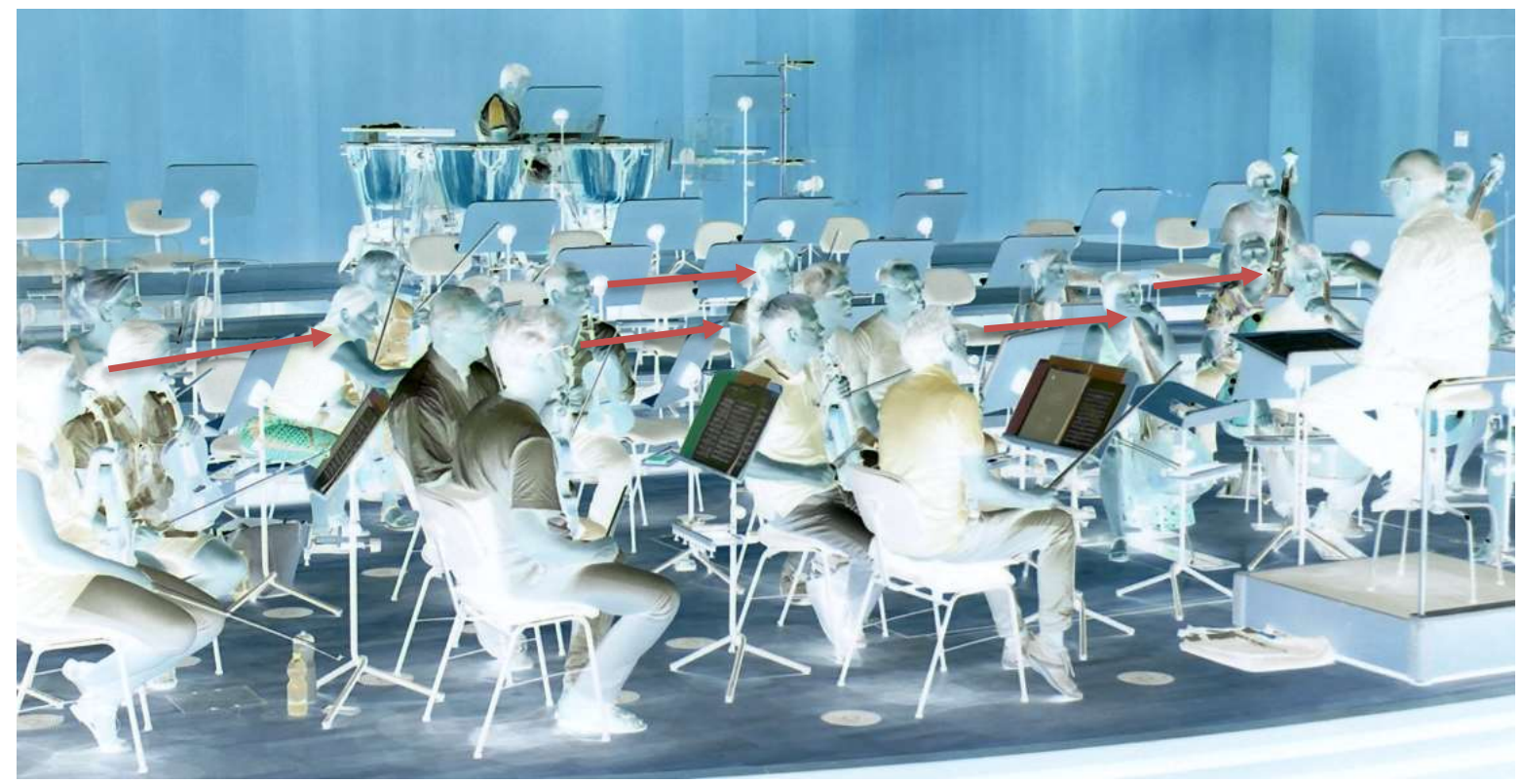

Abbildung 2: Aufmerksamkeit der Musiker/innen auf den Dirigenten gerichtet (ab Z09)

In Z09 kommt es außerdem zu einer Selbstreparatur des Dirigenten: Er unterbricht sich selbst sodann durch eine Verzögerungspartikel (eh) und nimmt die Einleitung der Erzählung durch das Personalpronomen io wiederum auf. Diese Art von ,change of footing“ (cf. Goffman 1981) schiebt die Verantwortung des Erzählten vom gesamten Orchester (noi) auf den Dirigenten alleine (io), letzterer wird zum principal, d. h. zu demjenigen, der verantwortlich für das Gesagte ist. Der Dirigent unterstützt die Einleitung seiner Erzählung durch die rechte Hand, die er vor der Brust zu einer Faust ballt. Die Hand bewegt sich leicht nach vorne und nach hinten, bis zum Ausdruck signore, bei dem sich die geballte Faust sichtlich nach vorne bewegt. ${ }^{10}$ Der Dirigent betont das Wort signore und verleiht ihm durch die Handbewegung nochmals Nachdruck. Auf diese Art und Weise etabliert er einen Referenten, der für seine folgende Erzählung wichtig sein wird:

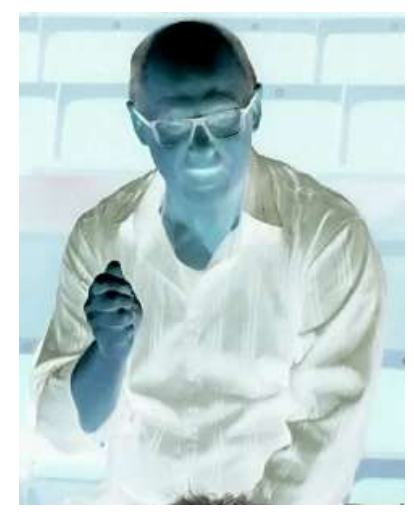

Abbildung 3: Dirigent bewegt rechte Faust nach vorne

In Z09-10 leitet der Dirigent durch den Ausdruck questa signore den Protagonisten (siehe $f$ gure nach Goffman 1981) seiner Erzählung ein. Während in Z04 noch von einem generischen Pianisten die Rede ist, kommt es in Z09-10 zu einer Spezifizierung des zum Thema stehenden

\footnotetext{
${ }^{10}$ Cf. hierzu auch die Taktstockgesten (oder beats) bei McNeill (1992) sowie Müller (1998), die im Allgemeinen dazu eingesetzt werden, das zugleich Gesagte hervorzuheben bzw. zu betonen (cf. auch Schoonjans 2018).
} 
Pianisten. Durch den Gebrauch des Demonstrativartikels questa signalisiert der Dirigent den Musiker/inne/n, dass sie die zur Sprache gebrachte Person kennen und in der Lage sind, diese zu identifizieren. In diesem Fall kann von einem „Auerismus“"11 (Lavric 2011: 70) gesprochen werden, ein Prozess, bei dem in einem Gespräch ein Demonstrativum als Indiz für Indexikalität fungiert. Der/die Sprecher/in, der/die das Demonstrativum benutzt, geht davon aus, dass sein/ihr Gesprächspartner/in in der Lage ist, den/die Referenten/in zu bestimmen. Das Demonstrativum kann von einer kurzen Beschreibung begleitet sein, auf die eine Pause folgt, in der das Gegenüber reagieren und eventuell eine genauere Beschreibung einfordern kann. Dieser Prozess setzt sich so lange fort, bis der/die Referent/in identifiziert wird.

In dem vorliegenden Beispiel führt der Dirigent seine Beschreibung durch ein Substantiv (signore) und ein Relativpronomen (che) weiter aus. Er setzt diese Charakterisierung allerdings nicht verbal fort, sondern er benutzt die rechte Hand, die er zum Gesicht führt. Damit nimmt er gestisch voraus, was er kurz darauf verbal anführen wird (sarà con noi in Z10 und Z12):

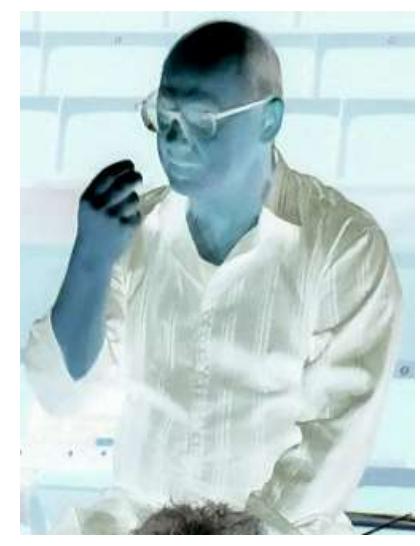

Abbildung 4: Dirigent führt rechte Hand zum Gesicht

An dieser Stelle schaltet sich der Konzertmeister, der links vom Dirigenten ganz außen sitzt, ein. In Z11 reagiert er mit einem zuerkennenden si auf den Turn des Dirigenten. Auch der Dirigent zeigt eine Reaktion seinerseits, indem er gleichzeitig zur Äußerung des Konzertmeisters in dessen Richtung blickt. Es bleibt bei diesem gestischen Aufzeigehandeln, das eine Wahrnehmung des Turns des Konzertmeisters anzeigt. In Z12 führt der Dirigent seinen begonnenen Satz - an das gesamte Orchester gerichtet - weiter.

Hier entstehen gleich zwei multimodale, zeitlich organisierte Gestalten, die sich durch das Zusammenspiel von Sprache, Körper, Raum und Zeit auszeichnen. Die erste multimodale Gestalt besteht darin, dass es in Z10 nach der Einleitung eines Relativsatzes (durch den Ausdruck che) zu einer (Zeige-)Geste kommt. Indem der Dirigent die rechte Hand zum Gesicht führt und damit auf sich selbst zeigt, führt er eine Referenzhandlung aus. Gleichzeitig nimmt diese Zeigegeste die Nennung des Deiktikums ([con] noi in Z12) vorweg. Den TRP (,transition relevant place“,

\footnotetext{
11 Der Begriff ist nach dem deutschen Linguisten Peter Auer benannt, der dieses Phänomen in mündlichen Kommunikationssituationen als erster für das Deutsche beschrieben hat (cf. Auer 1981, 1984). Auer (1981, 1984) zeigt, dass der Demonstrativbegleiter dieser von einem Sprecher/einer Sprecherin nicht nur benutzt werden kann, um anzuzeigen, dass der/die Referent/in dem/der Gesprächspartner/in bekannt sein dürfte, sondern auch um zu testen, ob geteiltes Wissen vorhanden ist, um den/die Referent/in zu identifizieren. Lavric (2011) untersucht dasselbe Phänomen für das Spanische und das Französische.
} 
Sacks/Schegloff/Jefferson 1974) in Form einer Pause am Ende von Z10 nutzt der Konzertmeister, um den Turn für sich zu beanspruchen und die Ausführungen des Dirigenten zu ratifizieren. Dafür kombiniert er den bejahenden Ausdruck sì mit einem Nicken und realisiert damit eine multimodale Gestalt, die nicht nur Verstehen signalisiert, sondern auch als „,continuer“ (Schegloff 1982) fungiert, der das Rederecht weiter dem Dirigenten zuweist. Genau in dem Moment der Äußerung des Konzertmeisters blickt der Dirigent in dessen Richtung und zeigt damit ,engagement" an (cf. Goffman 1971: 45-51). In Z12 wendet der Dirigent den Blick abermals dem Orchester zu und führt den eröffneten Relativsatz aus Z10 (che sarà) sprachlich zu Ende (con noi).

In Z13 meldet sich der Konzertmeister erneut zu Wort, durch ein weiteres zuerkennendes Signal $(\mathrm{mhm})$. Auf diesen Turn reagiert der Dirigent weder gestisch noch verbal - und signalisiert damit „disengagement“ (cf. Goffman 1971: 45-51) -, sondern er führt seine Aussage über den Pianisten weiter, indem er in Z14 mit der rechten Hand auf die Partitur tippt und damit - ähnlich wie in Z10 - durch eine Geste eine verbale Ausführung antizipiert (a suonare questo secondo premio di busoni in Z16). In Z15 gibt der Konzertmeister durch ein Detail (il secondo) zu verstehen, dass er in der Lage ist, den eingeführten Referenten (den Pianisten) zu identifizieren (cf. Lavric 2011: 79). Mit il secondo bezieht er sich auf den zweiten Preis eines internationalen Wettbewerbs für Pianisten/innen und nimmt praktisch die Identifizierung des Referenten vorweg. Außerdem passt dieses Detail genau in das, was der Dirigent erzählen möchte, nämlich, dass es beim letzten Auftritt des Orchesters mit dem Pianisten bei eben diesem Wettbewerb, bei dem er den zweiten Preis gewann, zu Schwierigkeiten kam (siehe Z17-18). In diesem Abschnitt gestalten der Dirigent und der Konzertmeister die Identifizierung eines Referenten gemeinsam auf ko-konstruierte (Dausendschön-Gay/Gülich/Krafft 2015) und inkrementelle (Schegloff 2016) Art und Weise. Der Dirigent nimmt ein vorgegebenes Inkrement (il secondo) des Konzertmeisters in Z17 auf. Im Anschluss daran richtet er den Blick ein weiteres Mal nach links zum Konzertmeister hin aus und verhaftet diesen dort bis zum Abschluss der Sequenz über den Pianisten und den Klavierwettbewerb (Z18). Der Konzertmeister zeigt durch ein Nicken an (siehe Z17), dass er die Rolle des (aufmerksamen) Zuhörers einnimmt und dass er dem Dirigenten in seiner Aussage zustimmt (era molto pericoloso).

In Z16 ist damit die Referenzialisierungssequenz abgeschlossen, ab Z17 leistet der Dirigent die eigentliche narrative Rekonstruktionsarbeit (cf. Sacks 1989). Diese wird von nonverbalen Elementen begleitet, die das Erzählte unterstützen und teilweise auch komplettieren. In Z20 streckt der Dirigent vier Finger der rechten Hand nach oben, bevor er vom quarto premio spricht:

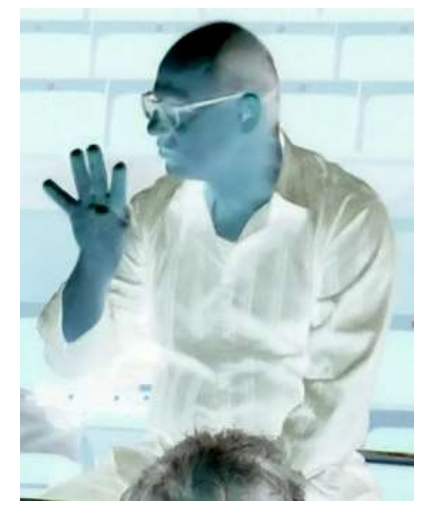

Abbildung 5: Dirigent streckt vier Finger der rechten Hand aus

ISSN 1615-3014 
Durch diese ikonische Geste ${ }^{12}$ nimmt er wie bereits in Z10 und Z14 seine kurz darauffolgende verbale Aussage voraus. Einen Unterschied zu Z10 und Z14 gibt es allerdings: In Z20 bereitet der Dirigent seine Geste während einer verbalen Aussage vor und führt sie fast simultan zur entsprechenden Aussage quarto premio aus. Dahingegen pausiert der Dirigent in Z10 und Z14 mit seinem Sprechen und gibt der Geste den Platz oder den Raum, dass die Aufmerksamkeit des Publikums sich einzig und allein auf die Geste konzentrieren kann. Bei Kendon (2004) gibt es eine ähnliche Kategorie an Gesten, bei denen ,speech is paused to allow a gesture to stand on its own“ (147). Die Geste in Z20 hingegen ist nicht mehr frei von Verbalität, sondern das Strecken der vier Finger dupliziert die verbale Aussage vielmehr (cf. Kendon 2004: 179). Hier hält der Dirigent seine Geste aber länger, auch noch in Z21, sobald er von einem weiteren musikalischen Wettbewerb spricht, und verleiht ihr dadurch Nachdruck.

Auf diese Aussage des Dirigenten reagiert in Z21 nicht nur der Konzertmeister durch ein weiteres zuerkennendes Nicken, sondern auch ein anderer Violinist, der in der ersten Reihe sitzt. Dieser weitet die Augen und öffnet den Mund:

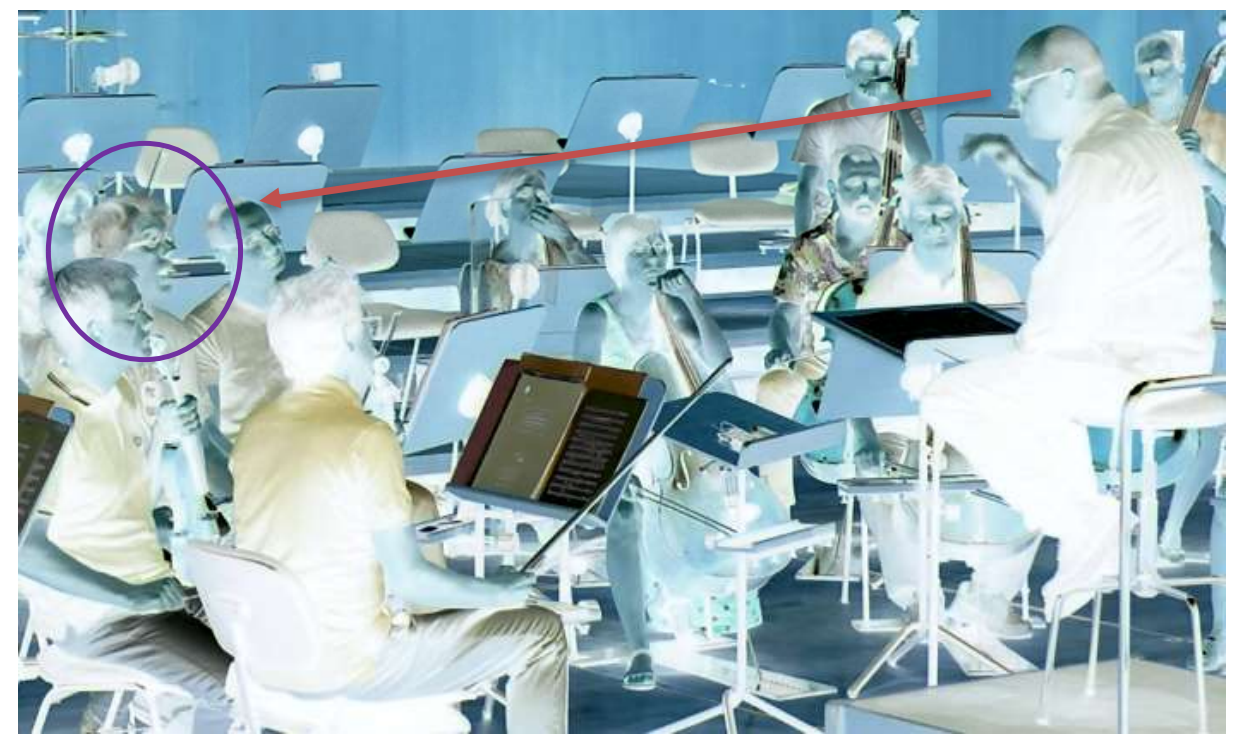

Abbildung 6: Blicklich-mimische Reaktion des Violinisten in der ersten Reihe

Durch diese blicklich-mimische Reaktion zeigt der Violinist nicht nur Erstaunen über die Erzählung des Dirigenten an, sondern er schlüpft - ähnlich wie der Konzertmeister in Z17 - in die Rolle des aufmerksamen Zuhörers. Interessant ist, dass der Dirigent ab Z20 in die Runde blickt, sein Blick allerdings just im Moment der multimodalen Reaktion des Violinisten auf diesem kurz haften bleibt (siehe Abbildung 6) und ihn dadurch in seiner Rolle als Zuhörer bestätigt. Hier entsteht eine weitere multimodale Gestalt, in der mimische, blickliche und räumliche Ressourcen zusammenspielen und sequenziell aufeinander abgestimmt sind.

Dieser Abschnitt zwischen Z19 und Z21 kann als Klimax der Erzählung aufgefasst werden. Der Dirigent setzt hier unterschiedliche Modalitäten ein, um den Höhepunkt der Geschichte vorzubereiten. Er benutzt unterschiedliche Blickrichtungen (Blick in die Runde vs. Blick nach links)

\footnotetext{
12 Es kann hier auch von einer emblematischen Geste (cf. Ekman/Friesen 1969) gesprochen werden. Embleme sind Gesten, die eine eingeschränkte und präzise Bedeutung haben und mit ein oder zwei Wörtern verbal übersetzt werden können, wie in diesem Fall die Zahl vier.
} 
und hebt sowohl prosodisch (mesi in Z19, quarto in Z20, elisabetta in Z21) als auch gestisch (quarto in Z20) bestimmte Ausdrücke hervor. Außerdem interagieren während dieser Sequenz der Violinist (V2) in der ersten Reihe und der Konzertmeister durch mimische und gestische Zeichen mit dem Dirigenten. Bei dieser Klimax geschieht also sichtbar und hörbar mehr als z. B. in der Einleitungssequenz: ${ }^{13}$ Der Dirigent als Erzähler und die Musiker/innen als Zuhörer/innen gestalten die Dramatisierung der Narration auf interaktive und multimodale Art und Weise.

Auffallend ist außerdem, dass der Dirigent den Blick während der Erzählung immer wieder zum Konzertmeister richtet, etwa in Z11, Z17 und Z19. Der Konzertmeister ist der Vertreter des gesamten Orchesters und erster Ansprechpartner für den Dirigenten in musikalischen Fragen. Auch für die Erzählung des Dirigenten entpuppt er sich als - scheinbar - vorrangiger Empfänger. Das Teilnehmerformat verschiebt sich immer wieder kurz vom gesamten Orchester auf den Dirigenten und den Konzertmeister, es kommt zu einer dyadischen Konstellation bzw. zu einer one-face-to-one-face-Interaktion. Diese Konstellation ist allerdings immer nur von kurzer Dauer, bevor die vorherrschende one-face-to-many-faces-Interaktion wieder aufgenommen wird.

Bevor der Dirigent zum Ende seiner Geschichte kommt, beginnt er in Z19 mit einem Imperativ in der ,wir'-Form (speriamo dopo...) und führt diesen nach einer Expansion (siehe Z19-21) in Z22 zu Ende. Hier wechselt der Dirigent von der, ich'-Form (siehe Z09) in die ,wir'-Form und schließt dadurch alle Beteiligten an der Interaktion mit in seine Aussage ein bzw. macht seine Aussage für alle Anwesenden relevant (cf. Lerner/Kitzinger 2007). An dieser Stelle setzt bereits ein pre-ending der Erzählung ein: in Z17-18 spricht der Dirigent noch in der Vergangenheit (era) - wie es für eine Geschichte durchaus typisch ist (cf. z. B. Gülich 2004) -, in Z19 leitet er einen Satz durch den Imperativ speriamo ein, der sich auf die Aufführung in den kommenden Tagen bezieht. In Z22 führt der Dirigent diesen Satz im Präsens (lui è un po ' maturizzato) zu Ende: Die Pointe der Geschichte ist nun erreicht.

Für die Pointe realisiert der Dirigent eine multimodale Gestalt, die darin besteht, dass der Ausdruck maturizzato prosodisch hervorgehoben und durch eine Geste begleitet wird. Der Dirigent bewegt beide Hände mit den Handflächen nach oben vor der Brust leicht nach oben und nimmt gleichzeitig die Schultern mit:

\footnotetext{
${ }^{13}$ Cf. auch König/Oloff (2018a: 220): „Besonders sicht- und hörbar in Erzählungen ist die Klimax, die durch eine Anhäufung und Intensivierung verbaler, vokaler und visueller Ressourcen aus der Gesamterzählung heraussticht, auch sind hiernach besondere Rezipientenreaktionen erwartbar“.
} 


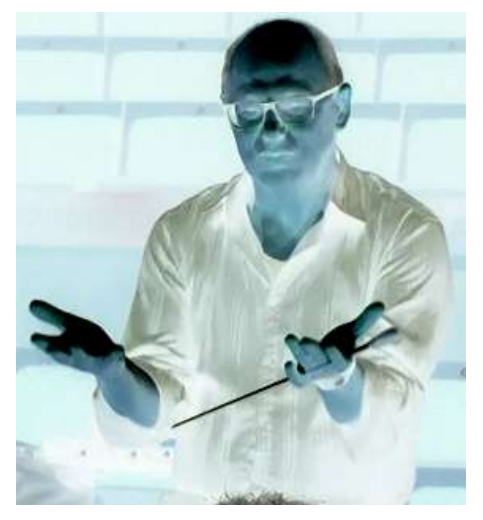

Abbildung 7: Dirigent bewegt beide Hände auf Brusthöhe

Der Dirigent verknüpft hier den Ausdruck maturizzato (,gereift") mit einer Hand- und Schulterbewegung nach oben. Auf diese Weise illustriert er gestisch (und auch prosodisch), dass die Reifung des Pianisten nun (hoffentlich) vorangeschritten ist und der Auftritt dieses Mal ohne Probleme über die Bühne gehen kann.

Auf den Höhepunkt der Erzählung reagieren die Musiker/innen bereits währenddessen mit einem Lachen (siehe Z22), das sie auch im Anschluss daran fortsetzen (siehe Z23). In Z24 wiederholt die Bratschistin, die in der ersten Reihe ganz rechts außen sitzt, den Ausdruck des Dirigenten am Ende seiner Erzählung (maturizzato). Dabei lächelt sie und blickt zum Dirigenten (der Dirigent erwidert diesen Blick in Z25, siehe Blick nach rechts). Diese Reaktionen können als Antwortsequenz (cf. Sacks 1989) bezeichnet werden: Die Rezipienten/innen reagieren mimisch und verbal auf das Erzählte und geben damit auch gleichzeitig einen indirekten Kommentar zur Geschichte ab: Sie drücken Komplizität mit dem Dirigenten aus.

Interessant ist, dass der Dirigent nach seiner Erzählung den Musiker/inne/n Zeit gibt, um darauf reagieren zu können. Er spricht nicht weiter, sondern hält sich durch ein Lächeln (siehe Z2324) sowie durch eine körperliche Bewegung (siehe das Aufstehen und Hinsetzen in Z25) im Hintergrund. Damit projiziert er bzw. setzt eine bestimmte sichtbare (oder auch hörbare) Antwort seiner Rezipienten/innen relevant (cf. Ruusuvuori/Peräkylä 2009).

Das Aufstehen und das Hinsetzen des Dirigenten sowie der Blick auf die Partitur in Z25 zeigen an, dass die Erzählsequenz nun abgeschlossen ist und dass ein Übergang zu einer anderen Aktivität erfolgen wird. Der Dirigent lokalisiert eine bestimmte Stelle in der Partitur (due in Z25) und spezifiziert damit eine Einsatzstelle (cf. Schmidt 2014), ab der die Musiker/innen erneut spielen sollen.

Damit ist die Erzählung des Dirigenten innerhalb instruktiver Handlungen eingebettet. In Z0108 instruiert der Dirigent, wie eine bestimmte Stelle in der Partitur (secondo dopo tre) zu spielen ist (subito più mosso), in Z25 weist er an, ab wo erneut gespielt werden soll. Inmitten dieser zusammenhängenden Instruktionssequenz kommt es in Z09-Z24 zu einer narrativen Rekonstruktion. Diese steht zwar in Verbindung zu dem geprobten Stück, allerdings impliziert sie keinerlei instruktiven, sondern allenfalls einen humoristischen oder auflockernden Charakter. 


\section{$5 \quad$ Fazit}

Aus dem analysierten Beispiel für narrative Einheiten in der Orchesterprobe geht hervor, dass sich das Erzählen - ähnlich wie in einem Alltagsgespräch - aus anderen konversationellen Aktivitäten heraus entwickelt. In dem speziellen Fall der Orchesterprobe sind das die Anweisungen und Anmerkungen des Dirigenten zur Musik, d. h. die Narration wird in eine solch anleitende Sequenz eingebettet. Die Erzählung selbst nutzt der Dirigent, um eine Erfahrung wiederzugeben und die Probe gleichzeitig durch einen kleinen Scherz aufzuheitern.

In seiner narrativen Einheit realisiert der Dirigent die Teilaufgaben der narrativen Rekonstruktion oder Jobs (cf. Hausendorf/Quasthoff 1996, 2005). Er beginnt mit der Etablierung des Erzählens als Aktivität (inhaltliche bzw. formale Relevanzsetzung). Dann thematisiert er einen Referenten und ein Ereignis, bevor er die eigentliche narrative Einheit durchführt. Nach einer Pointe schließt er die Narration ab, indem er seinen Zuhörern/innen Raum für eine Reaktion bietet und schließlich zum nicht-narrativen Gespräch überleitet. Diese einzelnen Erzählschritte sind vergleichbar mit den ,storytelling sequences“ nach Sacks (1989), und zwar die Einleitungssequenz, die eigentliche Erzählung, sowie die Antwort- und Bearbeitungssequenz.

Dabei läuft die narrative Rekonstruktionsarbeit multimodal und interaktiv ab. Der Dirigent verwendet für sein narratives Gefüge zahlreiche, unterschiedlichste multimodale Ressourcen, die von Gestik und Mimik bis hin zu Blicken und Körperbewegungen reichen. Während seiner Narration geht er außerdem auf Kommentare des Konzertmeisters und auf die mimischen Reaktionen der Musiker/innen ein und gestaltet seine Geschichte so, dass sie empathisch wirkt. Dabei entstehen multimodale, sequenziell organisierte Gestalten, die zum interaktiven und multimodalen Charakter von Erzählungen und darüber hinaus zum Erreichen von Intersubjektivität in der Orchesterprobe beitragen - ein übergeordnetes Ziel, das die Arbeit zwischen Dirigent/in und Musiker/inne/n in der Orchesterprobe beherrscht.

Die vorliegende Studie liefert damit einen Beitrag zur holistischen Auffassung von Erzählen (cf. König/Oloff 2018a), bei der die Rollen von Erzähler/in und Zuhörer/in in den Hintergrund rücken. Sie veranschaulicht generische Aspekte des multimodalen und interaktiven Erzählens in der Orchesterprobe, die als soziale Praktiken eingestuft werden können. Dabei steht allerdings nur eine Funktion des Erzählens (Scherzen/Auflockern) im Fokus, die es durch weitere (etwa Veranschaulichen, Argumentieren) in vergleichbaren Studien zu ergänzen gilt.

\section{Literaturverzeichnis}

Auer, Peter (1981): „Zur indexikalitätsmarkierenden Funktion der demonstrativen Artikelform in deutschen Konversationen“. In: Hindelang, Götz/Zillig, Werner (eds.): Sprache: Verstehen und Handeln. Akten des 15. Linguistischen Kolloquiums, Münster 1980. 2. Bd. Tübingen, Niemeyer: 301-310.

Auer, Peter (1984): „Referential problems in conversation“. Journal of Pragmatics 8: 627-648.

Bamberg, Michael/Georgakopoulou, Alexandra (2008): „Small Stories as a New Perspective in Narrative and Identity Analysis". Text \& Talk 28/3: 377-396.

Becker-Mrotzek, Michael (2011): „Der Erzählkreis als Exempel für die Besonderheiten der Unterrichtskommunikation“. In: Bräuer, Christoph/Ossner, Jakob (eds.): Kommunikation 
und Interaktion im Unterricht (OBST: Osnabrücker Beiträge zur Sprachtheorie 80). Duisburg, Universitätsverlag Rhein-Ruhr: 31-47.

Bergmann, Jörg/Luckmann, Thomas (1995): „Reconstructive genres of everyday communication”. In: Quasthoff, Uta M. (ed.): Aspects of Oral Communication. Berlin/New York, de Gruyter: 289-304.

Birkner, Karin (2017): „Erzählen im Arzt/Patient-Gespräch“. In: Huber, Martin/Schmid, Wolf (eds.): Grundthemen der Literaturwissenschaft: Erzählen. Berlin/Boston, de Gruyter: 547-566.

Bonu, Bruno (1998): „Narration et interaction“. In: Desgoutte, Jean-Paul (ed.): Les figures du sujet en sciences humaines: motifs de rupture. Paris, L'Harmattan: 203-221.

Boyes-Bräm, Penny/Bräm, Thüring (2000): „A Pilot Study of the Expressive Gestures Used by Classical Orchestra Conductors". In: Emmorey, Karen/Lane, Harlan (eds.): The Signs of Language Revisited. Mahwah/New Jersey, Lawrence Erlbaum: 143-167.

Boyes-Bräm, Penny/Bräm, Thüring (2004): „Expressive Gestures used by Classical Orchestra Conductors". In: Müller, Cornelia/Posner, Roland (eds.): The Semantics and Pragmatics of Everyday Gestures. Berlin, Weidler:127-143.

Clark, Herbert H. (1996): Using Language. Cambridge: CUP.

Dausendschön-Gay, Ulrich/Gülich, Elisabeth/Krafft, Ulrich (2015): „Zu einem Konzept von Ko-Konstruktion“. In: Dausendschön-Gay, Ulrich/Gülich, Elisabeth/Krafft, Ulrich (eds.): Ko-Konstruktionen in der Interaktion. Die gemeinsame Arbeit an Äußerungen und anderen sozialen Ereignissen. Bielefeld, transcript: 21-36.

De Fina, Anna/Georgakopoulou, Alexandra (2012): Analyzing Narrative: Discourse and Sociolinguistic Perspectives. Cambridge: Cambridge University Press.

De Fina, Anna/Georgakopoulou, Alexandra (eds.) (2015): Handbook of narrative analysis. Chichester: Blackwell.

Deppermann, Arnulf (2008): Gespräche analysieren. Eine Einführung. 4. Auflage. Wiesbaden: Verlag für Sozialwissenschaften.

Deppermann, Arnulf (2018): „Sprache in der multimodalen Interaktion“. In: Deppermann, Arnulf/Reineke, Silke (eds.): Sprache im kommunikativen, interaktiven und kulturellen Kontext. Berlin/Boston, de Gruyter: 51-85.

Drew, Paul/Heritage, John (eds.) (1992): Talk at work. Interaction in institutional settings. Cambridge: CUP.

Ehlich, Konrad (ed.) (1984): Erzählen in der Schule. Tübingen: Narr.

Ekman, Paul/Friesen, Wallace V. (1969): „The Repertoire of Nonverbal Behavior. Categories, Origins, Usage, and Coding“. Semiotica 1: 49-98.

Goffman, Erving (1971): Verhalten in sozialen Situationen. Strukturen und Regeln der Interaktion im öffentlichen Raum. Gütersloh: Bertelsmann.

Goffman, Erving (1979): „Footing“. Semiotica 25/1/2: 1-29.

Goffman, Erving (1981): Forms of talk. Oxford: Blackwell.

Goodwin, Charles (1984): „Notes on story structure and the organization of participation”. In: Atkinson, J. Maxwell (ed.): Structures of Social Action. Studies in Conversation Analysis. Cambridge, CUP: 225-246.

Goodwin, Charles (1994): „Professional Vision“. American Anthropologist 96/3: 606-633. 
Goodwin, Charles (2007): „Participation, stance and affect in the organization of activities”. Discourse \& Society 18/1: 53-73.

Goodwin, Charles/Goodwin Marjorie H. (2004): „Participation“. In: Duranti, Alessandro (ed.): A Companion to Linguistic Anthropology. Malden/MA, Blackwell: 222-244.

Gülich, Elisabeth (2004): Erzählen aus konversationsanalytischer Perspektive: Versuch einer Synthese. (Unveröffentlichtes Grundlagenpapier für die Sommerakademie "Narrative Sinnbildung" an der Universität Greifswald, 30.08. - 11.09.2004). Greifswald.

Gülich, Elisabeth (2008): „Alltägliches erzählen und alltägliches Erzählen“. Zeitschrift für germanistische Linguistik 36: 403-426.

Gülich, Elisabeth/Mondada, Lorenza (2008): Konversationsanalyse. Eine Einführung am Beispiel des Französischen. Tübingen: Niemeyer.

Gülich, Elisabeth/Schöndienst, Martin/Surmann, Volker (2003): „Schmerzen erzählen Geschichten - Geschichten erzählen Schmerzen“. Psychotherapie und Sozialwissenschaft 5/3: 220-249.

Hausendorf, Heiko/Quasthoff, Uta M. (1996): Sprachentwicklung und Interaktion. Eine linguistische Studie zum Erwerb von Diskursfähigkeiten. Opladen: Westdeutscher Verlag.

Hausendorf, Heiko/Quasthoff, Uta M. (2005): „Konversations-/Diskursanalyse“. (Sprach-)Entwicklung durch Interaktion. In: Mey, Günter (ed.): Qualitative Forschung in der Entwicklungspsychologie. Köln, Kölner Studien Verlag: 585-618.

Haviland, John B. (2007): „Master Speakers, Master Gestures: A String Quartet Master Class“. In: Duncan, Susan D./Cassell, Justine/Levy, Elena T. (eds.): Gesture and the Dynamic Dimension of Language: Essays in Honor of David McNeill. Amsterdam/Philadelphia, Benjamins: $147-172$.

Hoffmann, Lothar (1980): „Zur Pragmatik von Erzählungen vor Gericht“. In: Ehlich, Konrad (ed.): Erzählen im Alltag. Frankfurt a. M., Suhrkamp: 28-63.

Jefferson, Gail (1978): „Sequential aspects of storytelling in conversation”. In: Schenkein, Jim (ed.): Studies in the organization of conversational interaction. New York, Academic Press: 219-248.

Kallmeyer, Werner (1981): „Gestaltungsorientiertheit in Alltagserzählungen“. In: Kloepfer, Rolf/Janetzke-Dillner, Gisela (eds.): Erzählung und Erzählforschung im 20. Jahrhundert. Stuttgart, Kohlhammer: 409-429.

Kendon, Adam (2004): Gesture: Visible Action as Utterance. Cambridge: Cambridge University Press.

König, Katharina/Oloff, Florence (2018a): „Ansätze zu einer multimodalen Erzählanalyse. Einführung in das Themenheft". In: König, Katharina/Oloff, Florence (eds.): Themenheft Erzählen multimodal. Gesprächsforschung - Online-Zeitschrift zur verbalen Interaktion 19: 207-241.

König, Katharina/Oloff, Florence (2018b): „Die Multimodalität alltagspraktischen Erzählens“، Zeitschrift für Literaturwissenschaft und Linguistik 48/2: 277-307.

Kotthoff, Helga (2015): „Narrative constructions of school-oriented parenthood during parentteacher-conferences". Linguistics and Education 31: 286-303.

Kotthoff, Helga (2017): „Erzählen in Gesprächen. Eine Einführung in die konversationsanalytische Erzählforschung mit Übungsaufgaben“. Freiburger Arbeitspapiere zur Germanistischen Linguistik 38: 1-77. 
Kotthoff, Helga (2018): „Nicht ausgebaute Erzählungen“. In: Zeitschrift für Literaturwissenschaft und Linguistik 48/2: 225-248.

Labov, William/Waletzky, Joshua (1967): „Narrative analysis: oral versions of personal experience". In: Helm, June (eds): Essays on the verbal and visual arts. Seattle, University of Washington Press: 12-44.

Lavric, Eva (2011): „La chica esa - ton collègue là. Les Auérismes, ou : Comment co-construire les référents dans la conversation (version longue)“. In: Brzozowska-Zburzyńska, Beata/Posturzyńska-Bosko, Małgorzata (eds.): Expression indexicale. Lublin, Wydawnictwo Uniwersytetu Marii Curie-Skłodowskiej: 65-82.

Leodolter, Ruth (1975): Das Sprachverhalten von Angeklagten bei Gericht. Ansätze zu einer soziolinguistischen Theorie der Verbalisierung. Regensburg: Scriptor.

Lerner, Gene H./Kitzinger, Celia (2007): „Extraction and aggregation in the repair of individual and collective self-reference“. Discourse Studies 9/4: 526-557.

Lucius-Hoene, Gabriele/Deppermann, Arnulf (2002): Rekonstruktion narrativer Identität. Ein Arbeitsbuch zur Analyse narrative Interviews. Opladen: Leske \& Budrich.

Mandelbaum, Jenny (2013): „Storytelling in conversation“. In: Sidnell, Jack/Stivers, Tanya (eds.): The Handbook of Conversation Analysis. Chichester etc., Wiley-Blackwell: 492-507.

Martens-Schmid, Karin (2000): „Sprache, Affekt und Handeln“. In: Kruse, Gunther/Gunkel, Stefan (eds.): Sprache und Handeln - Was bewirkt die Wirklichkeit? Hannover, Hannoversche Ärzte-Verlags-Union: 200-228.

Martínez, Matías (ed.) (2017): Erzählen. Ein interdisziplinäres Handbuch. Stuttgart: Metzler.

McNeill, David (1992): Hand and Mind: What gestures reveal about thought. Chicago: University of Chicago Press.

Merlino, Sara (2014): „Singing in ,Another' Language: How Pronunciation Matters to the Organization of Choral Rehearsals". Social Semiotics 24/4: 420-445.

Mondada, Lorenza (2014a): Conventions for multimodal transcription. franzoesistik.philhist. unibas.ch/fileadmin/user_upload/franzoesistik/mondada_multimodal_conventions.pdf [26.10.2020].

Mondada, Lorenza (2014b): „The local constitution of multimodal resources for social interaction". Journal of Pragmatics 65: 137-156.

Mondada, Lorenza (2016): „Challenges of multimodality: Language and the body in social interaction”. Journal of Sociolinguistics 20/2: 2-32.

Morris, Lawrence D. (1995): Testament. A Conduction Collection. (Notes to the 10-CD box set Testament). New York, New World Records (Recorded Anthology of American Music): $3-12$.

Morris, Lawrence D. (2006): „Conduction is (Link between Notation and Improvisation)“. In: Contemporary Music Review 25/5-6: 533-535.

Müller, Cornelia (1998): Redebegleitende Gesten. Kulturgeschichte - Theorie - Sprachvergleich. Berlin: Arno Spitz.

Niemelä, Maarit (2010): „The reporting space in conversational storytelling: Orchestrating all semiotic channels for taking a stance". Journal of Pragmatics 42: 3258-3270.

Niemelä, Maarit (2011): Resonance in Storytelling: Verbal, Prosodic and Embodied Practices of Stance Taking. Oulu B 95. researchgate.net/publication/278357795_RESONANCE_IN_ 
STORYTELLING_VERBAL_PROSODIC_AND_EMBODIED_PRACTICES_OF_ STANCE_TAKING [26.10.2020].

Ohlus, Sören (2014): Erzählen als Prozess. Interaktive Organisation und narrative Verfahren in mündlichen Erzählungen von Grundschulkindern. Tübingen: Stauffenburg.

Ohlus, Sören (2016): „Narrative Verfahren und mediale Bedingungen im mündlichen und schriftlichen Erzählen“. In: Behrens, Ulrike/Gätje, Olaf (eds.): Mündliches und schriftliches Handeln im Deutschunterricht. Wie Themen entfaltet werden. Frankfurt a. M. etc., Lang: $39-65$.

Oloff, Florence/König, Katharina (2018): „Zur Theatralität und Multimodalität des Erzählens in der Fernseh-Unterhaltung“. In: König, Katharina/Oloff, Florence (eds.): Themenheft Erzählen multimodal. Gesprächsforschung - Online-Zeitschrift zur verbalen Interaktion 19: 346-386.

Poggi, Isabella (2002): „The Lexicon of the Conductor's Face“. In: McKevitt, Paul/O’Nuallàin, Seán/Mulvihill, Conn (eds.): Language, Vision, and Music. Selected Papers from the $8^{\text {th }}$ International Workshop on the Cognitive Science of Natural Language Processing, Galway, 1999. Amsterdam/Philadelphia, Benjamins: 271-284.

Poggi, Isabella (2011): „Music and leadership: the Choir Conductor's multimodal communication”. In: Stam, Gale/Ishino, Mika (eds.): Integrating Gestures. The interdisciplinary nature of gestures. Amsterdam/Philadelphia, Benjamins: 341-353.

Quasthoff, Uta M. (1980): Erzählen in Gesprächen. Linguistische Untersuchungen zu Strukturen und Funktionen am Beispiel einer Kommunikationsform des Alltags. Tübingen: Narr.

Quasthoff, Uta M. (2001): „Erzählen als interaktive Gesprächsstruktur“. In: Brinker, Klaus et al. (eds.): Text- und Gesprächslinguistik. Ein internationales Handbuch zeitgenössischer Forschung. Berlin/New York, de Gruyter: 1293-1309.

Quasthoff, Uta M./Becker, Tabea (eds.) (2005): Narrative Interaction. Amsterdam/Philadelphia: Benjamins.

Rehbein, Jochen (1980): „Sequentielles Erzählen. Erzählstrukturen von Immigranten bei Sozialberatungen in England“. In: Ehlich, Konrad (ed.): Erzählen im Alltag. Frankfurt a. M., Suhrkamp: 64-108.

Ruusuvuori, Johanna/Peräkylä, Anssi (2009): „Facial and Verbal Expressions in Assessing Stories and Topics". Research on Language and Social Interaction 42/4: 377-394.

Sacher, Julia/Knerich, Heike/Lingnau, Beate (2015): „(Ko?-)Konstruktion in institutionellen Settings“. In: Dausendschön-Gay, Ulrich/Gülich, Elisabeth/Krafft, Ulrich (eds.): Ko-Konstruktionen in der Interaktion. Die gemeinsame Arbeit an Äußerungen und anderen sozialen Ereignissen. Bielefeld, transcript: 349-372.

Sacks, Harvey (1971): „Das Erzählen von Geschichten innerhalb von Unterhaltungen“. In: Kjolseth, Ralf/Sack, Fritz (eds.): Zur Soziologie der Sprache (Kölner Zeitschrift für Soziologie und Sozialpsychologie, Sonderheft 15): 307-314.

Sacks, Harvey (1989): „An analysis of the course of a joke's telling in conversation”. In: Baumann, Richard/Sherzer, Joel (eds.): Explorations in the ethnography of speaking. Cambridge: Cambridge University Press: 337-353.

Sacks, Harvey (1992): Lectures on conversation. Edited by Gail Jefferson, with an Introduction by Emanuel A. Schegloff. Vol. II. Oxford: Blackwell. 
Sacks, Harvey/Schegloff, Emanuel A./Jefferson, Gail (1974): „A Simplest Systematics of the Organization of Turn-Taking in Conversation“. Language 50/4: 696-735.

Schegloff, Emanuel A. (1982): „Discourse as an interactional achievement: Some uses of 'uh huh' and other things that come between sentences“. In: Tannen, Deborah (ed.): Analyzing discourse: Text and talk. Washington DC, Georgetown University Press: 71-93.

Schegloff, Emanuel A. (1997): „,Narrative analysis“ thirty years later“. In: Bamberg, Michael (ed.): Oral versions of personal experience: Three decades of narrative analysis. The Journal of Narrative and Life History 7/1-4: 97-106.

Schegloff, Emanuel A. (2016): „Increments“. In: Robinson, Jeffrey D. (ed.): Accountability in social interaction. New York, Oxford University Press: 239-263.

Schoonjans, Steven (2018): Modalpartikeln als multimodale Konstruktionen. Eine korpusbasierte Kookkurrenzanalyse von Modalpartikeln und Gestik im Deutschen. Berlin/Boston: de Gruyter.

Schmidt, Axel (2014): Spiel oder nicht Spiel? Zur interaktiven Organisation von Übergängen zwischen Spielwelt und Realwelt in Theaterproben. Mannheim: Verlag für Gesprächsforschung. verlag-gespraechsforschung.de/2014/axelschmidt.html [22.05.2020].

Schwarze, Cordula (2019): „Erzählen fürs Argumentieren. Das Verhältnis von Narration und Argumentation in Konfliktbearbeitungen im Gespräch“. Zeitschrift für Literaturwissenschaft und Linguistik 49: 51-70.

Selting, Margret et al. (2009): „Gesprächsanalytisches Transkriptionssystem (GAT 2)“. In: Gesprächsforschung - Online-Zeitschrift zur verbalen Interaktion 10: 353-402. gespraechsforschung-ozs.de/heft2009/px-gat2.pdf [19.05.2020].

Steensig, Jakob (2013): „Conversation analysis and affiliation and alignment”. In: Chapelle, Carol A. (ed.): The Encyclopedia of Applied Linguistics. Hoboken/NJ, Wiley-Blackwell: 944-948.

Stivers, Tanya (2008): „Stance, alignment and affiliation during story telling. When nodding is a token of premiminary affiliation“. Research on Language in Social Interaction 41: 29-55. Veronesi, Daniela (2014): „Correction Sequences and Semiotic Resources in Ensemble Music Workshops: The Case of Conduction ${ }^{\circledR} "$. Social Semiotics 24/4: 467-493.

Weeks, Peter (1996): „A rehearsal of a Beethoven passage: An analysis of correction talk”. Research on Language and Social Interaction 29: 247-290. 